\title{
Model-informed COVID-19 vaccine prioritization strategies by age and serostatus
}

\author{
Kate M. Bubar ${ }^{1,2 *}$, Kyle Reinholt ${ }^{3}$, Stephen M. Kissler ${ }^{4}$, Marc Lipsitch $^{4,5}$, Sarah Cobey $^{6}$, Yonatan H. Grad ${ }^{4}$, Daniel B. Larremore ${ }^{3,7 *}$ \\ ${ }^{1}$ Department of Applied Mathematics, University of Colorado Boulder, Boulder, C0, 80303, USA. ${ }^{2}$ IQ Biology Program, University of Colorado Boulder, Boulder, C0, 80309 , \\ USA. ${ }^{3}$ Department of Computer Science, University of Colorado Boulder, Boulder, C0, 80309, USA. “Department of Immunology and Infectious Diseases, Harvard T.H. Chan \\ School of Public Health, Boston, MA, 02115, USA. ${ }^{5}$ Center for Communicable Disease Dynamics, Harvard T.H. Chan School of Public Health, Boston, MA, 02115, USA. \\ ${ }^{6}$ Department of Ecology and Evolution, University of Chicago, Chicago, IL, 60637, USA. ${ }^{7 B i o F r o n t i e r s ~ I n s t i t u t e, ~ U n i v e r s i t y ~ o f ~ C o l o r a d o ~ B o u l d e r, ~ B o u l d e r, ~ C 0, ~ 80303, ~ U S A . ~}$
}

*Corresponding author. E-mail: kate.bubar@colorado.edu (K.M.B.); daniel.larremore@colorado.edu (D.B.L.)

Limited initial supply of SARS-CoV-2 vaccine raises the question of how to prioritize available doses. Here, we used a mathematical model to compare five age-stratified prioritization strategies. A highly effective transmission-blocking vaccine prioritized to adults ages 20-49 years minimized cumulative incidence, but mortality and years of life lost were minimized in most scenarios when the vaccine was prioritized to adults over 60 years old. Use of individual-level serological tests to redirect doses to seronegative individuals improved the marginal impact of each dose while potentially reducing existing inequities in COVID-19 impact. While maximum impact prioritization strategies were broadly consistent across countries, transmission rates, vaccination rollout speeds, and estimates of naturally acquired immunity, this framework can be used to compare impacts of prioritization strategies across contexts.

SARS-CoV-2 has caused a public health and economic crisis worldwide. As of January 2021, there have been over 85 million cases and 1.8 million deaths reported (I). To combat this crisis, a variety of non-pharmaceutical interventions have been implemented, including shelter-in-place orders, limited travel, and remote schooling. While these efforts are essential to slowing transmission in the short term, long-term solutions-such as vaccines that protect from SARS-CoV-2 infection- remain urgently needed. The benefits of an effective vaccine for individuals and their communities have resulted in widespread demand, so it is critical that decision-making on vaccine distribution is well motivated, particularly in the initial phases when vaccine availability is limited (2).

Here, we employ a model-informed approach to quantify the impact of COVID-19 vaccine prioritization strategies on cumulative incidence, mortality, and years of life lost. Our approach explicitly addresses variation in three areas that can influence the outcome of vaccine distribution decisions. First, we consider variation in the performance of the vaccine, including its overall efficacy, a hypothetical decrease in efficacy by age, and the vaccine's ability to block transmission. Second, we consider variation in both susceptibility to infection and the infection fatality rate by age. Third, we consider variation in the population and policy, including the age distribution, age-stratified contact rates, and initial fraction of seropositive individuals by age, and the speed and timing of the vaccine's rollout relative to transmission. While the earliest doses of vaccines will be given to front-line health care workers under plans such as those from the COVAX initiative and the US NASEM recommendations (3), our work is focused on informing the prioritization of the doses that follow. Based on regulatory approvals and initial vaccine rollout speeds of early 2021, our investigation focuses generally on scenarios with a partially mitigated pandemic ( $R$ between 1.1 and 2.0), vaccines with protective efficacy of $90 \%$, and rollout speeds of $0.2 \%$ of the population per day.

There are two main approaches to vaccine prioritization: (1) directly vaccinate those at highest risk for severe outcomes and (2) protect them indirectly by vaccinating those who do the most transmitting. Model-based investigations of the tradeoffs between these strategies for influenza vaccination have led to recommendations that children be vaccinated due to their critical role in transmission $(4,5)$ and have shown that direct protection is superior when reproduction numbers are high but indirect protection is superior when transmission is low (6). Similar modeling for COVID-19 vaccination has found that the optimal balance between direct and indirect protection depends on both vaccine efficacy and supply, recommending direct vaccination of older adults for low-efficacy vaccines and for high-efficacy but supply-limited vaccines (7). Rather than comparing prioritization strategies, others have compared hypothetical vaccines, showing that even those with lower efficacy for direct protection may be more valuable if they also provide better indirect protection by blocking transmission (8). Prioritization of transmission-blocking vaccines can also be dynamically updated based on the current state of the epidemic, shifting prioritization to avoid decreasing marginal returns (9). These efforts 
to prioritize and optimize doses complement other work showing that, under different vaccine efficacy and durability of immunity, the economic and health benefits of COVID-19 vaccines will be large in the short and medium terms (10). The problem of vaccine prioritization also parallels the more general problem of optimal resource allocation to reduce transmission, e.g., with masks (11).

\section{Evaluation of vaccine prioritization strategies}

We evaluated the impact of vaccine prioritization strategies using an age-stratified SEIR model, because age has been shown to be an important correlate of susceptibility (12-14), seroprevalence $(12,15)$, severity $(16-18)$, and mortality (19, 20 ). This model includes an age-dependent contact matrix, susceptibility to infection, and infection fatality rate (IFR), allowing us to estimate cumulative incidence of SARS-CoV-2 infections, mortality due to infection, and years of life lost (YLL) (supplementary materials, materials and methods) via forward simulations of one year of disease dynamics . Cumulative incidence, mortality, and YLL were then used as outcomes by which to compare vaccine prioritization strategies. These comparisons may be explored using accompanying open-source and interactive calculation tools that accompany this study.

We first examined the impact of five vaccine prioritization strategies for a hypothetical infection- and transmissionblocking vaccine of varying efficacy. The strategies prioritized vaccines to (1) children and teenagers, (2) adults between ages 20 and 49 years, (3) adults 20 years or older, (4) adults 60 years or older, and (5) all individuals (Fig. 1A). In all strategies, once the prioritized population was vaccinated, vaccines were allocated irrespective of age, i.e., in proportion to their numbers in the population. To incorporate vaccine hesitancy, at most $70 \%$ of any age group was eligible to be vaccinated (21).

We measured reductions in cumulative incidence, mortality, and YLL achieved by each strategy, varying the vaccine supply between $1 \%$ and $50 \%$ of the total population, under two scenarios. In Scenario 1, vaccines were administered to $0.2 \%$ of the population per day until supply was exhausted, with $R_{0}=1.15$, representing highly mitigated spread during vaccine rollout. In Scenario 2, vaccines were administered to $0.2 \%$ of the population per day until supply was exhausted, but with $R_{0}=1.5$, representing substantial viral growth during vaccine rollout (see Fig. 1 for example model outputs). Results for additional scenarios in which vaccines were administered before transmission began are described in Supplementary Text, corresponding to countries without ongoing community spread such as South Korea and New Zealand. We considered two ways in which vaccine efficacy (ve) could be below $100 \%$ : an all-or-nothing vaccine, where the vaccine provides perfect protection to a fraction ve of individuals who receive it, or as a leaky vaccine, where all vaccinated individuals have reduced probability ve of infection after vaccination (supplementary materials, materials and methods).

Of the five strategies, direct vaccination of adults over 60 years (60+) always reduced mortality and YLL more than the alternative strategies when transmission was high $\left[R_{0}=1.5\right.$; Scenario 2; 90\% efficacy (Fig. 1); 30\%-100\% efficacy (fig. S5)]. For lower transmission $\left(R_{0}=1.15\right.$; Scenario 1$)$, vaccination of adults 20-49 reduced mortality and YLL more than the alternative strategies, but differences between prioritization of adults 20-49, adults $20+$, and adults $60+$ were small for vaccine supplies above $25 \%$ (Fig. 1 and fig. S5). Prioritizing adults 20-49 minimized cumulative incidence in both scenarios for all vaccine efficacies (Fig. 1 and fig. S5). Prioritizing adults 2049 also minimized cumulative incidence in both scenarios under alternative rollout speeds $(0.05 \%$ to $1 \%$ vaccinated per day) (fig. S6). When rollout speeds were at least $0.3 \%$ per day and vaccine supply covered at least $25 \%$ of the population, the mortality minimizing strategy shifted from prioritization of ages 20-49 to adults $20+$ or adults $60+$ for Scenario 1; when rollout speeds were at least $0.75 \%$ per day and covered at least $24 \%$ of the population, the mortality minimizing strategy shifted from prioritization of adults $60+$ to adults $20+$ or $20-$ 49 for Scenario 2 (fig. S6). Findings for mortality and YLL were only slightly changed by modeling vaccine efficacy as all-or-nothing (fig. S5) or leaky (fig. S7).

\section{Impact of transmission rates, age demographics, and contact structure}

To evaluate the impact of transmission rates on the strategy that most reduced mortality, we varied the basic reproductive number $R_{0}$ from 1.1 to 2.0 when considering a hypothetical infection- and transmission-blocking vaccine with $90 \%$ vaccine efficacy. We found that prioritizing adults $60+$ remained the best way to reduce mortality and YLL for $R_{0} \geq 1.3$, but prioritizing adults 20-49 was superior for $R_{0} \leq$ 1.2 (Fig. 2, A and B, and fig. S8). Prioritizing adults 20-49 minimized infections for all values of $R_{0}$ investigated (fig. $\mathrm{S} 8$ ).

To determine whether our findings were robust across countries, we analyzed the ranking of prioritization strategies for populations with the age distributions and modeled contact structures of the United States, Belgium, Brazil, China, India, Poland, South Africa, and Spain. Across these countries, direct vaccination of adults $60+$ minimized mortality for all levels of vaccine supply when transmission was high $\left(R_{0}=1.5\right.$, Scenario 2) (Fig. 2E), but in only some cases when transmission was lower $\left(R_{0}=1.15\right.$, rollout $0.2 \%$ per day, Scenario 1) (Fig. 2D). Decreasing rollout speed from $0.2 \%$ to $0.1 \%$ per day caused prioritization of adults $60+$ to be favored in additional scenarios (Fig. 2C). Across countries, vaccination of adults 20-49 nearly always minimized infections, and 
vaccination of adults $60+$ nearly always minimized YLL for Scenario 2, but no clear ranking of strategies emerged consistently to minimize YLL in Scenario 1 (fig. S9).

\section{Vaccines with imperfect transmission blocking ef- fects}

We also considered whether the rankings of prioritization strategies to minimize mortality would change if a vaccine were to block COVID-19 symptoms and mortality with $90 \%$ efficacy but with variable impact on SARS-CoV-2 infection and transmission. We found that direct vaccination of adults $60+$ minimized mortality for all vaccine supplies and transmission-blocking effects under Scenario 2, and for all vaccine supplies when up to $50 \%$ of transmission was blocked in Scenario 1 (supplementary text and fig. S10).

\section{Variation in vaccine efficacy by age}

COVID-19 vaccines may not be equally effective across age groups in preventing infection or disease, a phenomenon known to affect influenza vaccines $(22-25)$. To understand the impact of age-dependent COVID-19 vaccine efficacy, we incorporated a hypothetical linear decrease from a baseline efficacy of $90 \%$ for those under 60 to $50 \%$ in those 80 and older (Fig. 3). As expected, this diminished the benefits of any prioritization strategy that included older adults. For instance, strategies prioritizing adults 20-49 were unaffected by decreased efficacy among adults $60+$, while strategies prioritizing adults $60+$ were markedly diminished (Fig. 3). Despite these effects, prioritization of adults $60+$ remained superior to the alternative strategies to minimize mortality in Scenario 2.

To test whether more substantial age-dependent vaccine effects would change which strategy minimized mortality in Scenario 2, we varied the onset age of age-dependent decreases in efficacy, the extent to which it decreased, and the baseline efficacy from which it decreased. We found that as long as the age at which efficacy began to decrease was 70 or older and vaccine efficacy among adults $80+$ was at least $25 \%$, prioritizing adults $60+$ remained superior in the majority of parameter combinations. This finding was robust to whether the vaccine was modeled as leaky vs all-or-nothing, but we observed considerable variation from country to country (fig. S11).

\section{Incorporation of population seroprevalence and in- dividual serological testing}

Due to early indications that naturally acquired antibodies correlate with protection from reinfection (26), seroprevalence will affect vaccine prioritization in two ways. First, depending on the magnitude and age distribution of seroprevalence at the time of vaccine distribution, the ranking of strategies could change. Second, distributing vaccines to seropositive individuals would reduce the marginal benefit of vaccination per dose.

To investigate the impact of vaccinating mid-epidemic while using serology to target the vaccine to seronegative individuals, we included age-stratified seroprevalence estimates in our model by moving the data-specified proportion of seropositive individuals from susceptible to recovered status. We then simulated two approaches to vaccine distribution. In the first, vaccines were distributed according to the five prioritization strategies introduced above, regardless of any individual's serostatus. In the second, vaccines were distributed with a serological test, such that individuals with a positive serological test would not be vaccinated, allowing their dose to be given to someone else in their age group .

We included age-stratified seroprevalence estimates from New York City [August 2020; overall seroprevalence 26.9\% (27)] and demographics and age-contact structure from the United States in evaluations of the previous five prioritization strategies. For this analysis, we focused on Scenario $2(0.2 \%$ rollout per day, $R_{0}=1.5$ inclusive of seropositives), and found that the ranking of strategies to minimize incidence, mortality, and YLL remained unchanged: prioritizing adults $60+$ most reduced mortality and prioritizing adults 20-49 most reduced incidence, regardless of whether vaccination was limited to seronegative individuals (Fig. 4). These rankings were unchanged when we used lower or higher age-stratified seroprevalence estimates to test the consistency of results (Connecticut, July 2020, overall seroprevalence 3.4\% (28) and synthetic, overall seroprevalence 39.5\%; Figs. S12 and S13). Despite lowered sensitivity to detect past exposure due to seroreversion $(29,30)$, preferentially vaccinating seronegative individuals yielded large additional reductions in cumulative incidence and mortality in locations with higher seroprevalence (Figs. 4 and fig. S13) and modest reductions in locations with low seroprevalence (fig. S12). These results remained unchanged when statistical uncertainty, due to sample size and imperfect test sensitivity and specificity, were incorporated into the model (31).

\section{Discussion}

This study demonstrated the use of an age-stratified modeling approach to evaluate and compare vaccine prioritization strategies for SARS-CoV-2. After accounting for countryspecific age structure, age-contact structure, infection fatality rates, and seroprevalence, as well as the age-varying efficacy of a hypothetical vaccine, we found that across countries those aged 60 and older should be prioritized to minimize deaths, assuming a return to high contact rates and pre-pandemic behavior during or after vaccine rollout. This recommendation is robust because of the dramatic differences in IFR by age. Our model identified three general regimes in which prioritizing adults aged $20-49$ would provide greater 
mortality benefits than prioritizing older adults. One such regime was in the presence of substantial transmission-mitigating interventions $\left(R_{0}=1.15\right)$ and a vaccine with $80 \%$ or higher transmission blocking effects. A second regime was characterized by substantial transmission-mitigating interventions $\left(R_{0}=1.15\right)$ and either rollout speeds of at most $0.2 \%$ per day or vaccine supplies of at most $25 \%$ of the population. The third regime was characterized by vaccines with very low efficacy in older adults, very high efficacy in younger adults, and declines in efficacy starting at age 59 or 69 . The advantage of prioritizing all adults or adults $20-49$ vs. adults $60+$ was small under these conditions. Thus, we conclude that for mortality reduction, prioritization of older adults is a robust strategy that will be optimal or close to optimal to minimize mortality for virtually all plausible vaccine characteristics.

In contrast, the ranking of infection-minimizing strategies for mid-epidemic vaccination led to consistent recommendations to prioritize adults 20-49 across efficacy values and countries. For pre-transmission vaccination, prioritization shifted toward children and teenagers for leaky vaccine efficacies $50 \%$ and below, in line with prior work (7), as well as for vaccines with weak transmission-blocking properties. Because a vaccine is likely to have properties of both leaky and all-or-nothing models, empirical data on vaccine performance could help resolve this difference in model recommendations, although data are difficult to obtain in practice [see, e.g., $(32,33)]$.

It is not yet clear whether the first-generation of COVID19 vaccines will be approved everywhere for the elderly or those under 18 (34-36). While our conclusions assumed that the vaccine would be approved for all age groups, the evaluation approaches introduced here can be tailored to evaluate a subset of approaches restricted to those within the age groups for which a vaccine is licensed, using open-source tools such as those that accompany this study. Furthermore, while we considered three possible goals of vaccinationminimizing cumulative incidence, mortality, or YLL-our framework can be adapted to consider goals such as minimizing hospitalizations, ICU occupancy (7) or economic costs (10).

We demonstrated that there is value in pairing individuallevel serological tests with vaccination, even when accounting for the uncertainties in seroprevalence estimates (31) and seroreversion (29). The marginal gain in effective vaccine supply, relative to no serological testing, must be weighed against the challenges of serological testing prior to vaccination. Serostatus itself is an imperfect indicator of protection, and the relationship of prior infection, serostatus, and protection may change over time $(10,26,29,30)$. Delays in serological tests results would impair vaccine distribution, but partial seronegative-targeting effects might be realized if those with past PCR-confirmed infections voluntarily deprioritized their own vaccinations.

The best performing strategies depend on assumptions about the extent of a population's interactions. We used prepandemic contact matrices (37), reflecting the goal of a return to pre-pandemic routines once a vaccine is available, but more recent estimates of age-stratified contact rates could be valuable in modeling mid-pandemic scenarios $(38,39)$. Whether pre-pandemic or mid-pandemic contact estimates are representative of contact patterns during vaccine rollout remains unknown and may vary based on numerous social, political, and other factors. The scenarios modeled here did not incorporate explicit non-pharmaceutical interventions, which might persist if vaccination coverage is incomplete, but are implicitly represented in Scenario $1\left(R_{0}=1.15\right)$.

Our study relies on estimates of other epidemiological parameters. In local contexts, these include age-structured seroprevalence and IFR, which vary by population $(19,20,40)$. Globally, key parameters include the degree to which antibodies protect against reinfection or severity of disease and relative infectiousness by age. From vaccine trials, we also need evidence of efficacy in groups vulnerable to severe outcomes, including the elderly. Additionally, it will be critical to measure whether a vaccine that protects against symptomatic disease also blocks infection and transmission of SARSCoV-2 (41).

The role of children during this pandemic has been unclear. Under our assumptions about susceptibility by age, children are not the major drivers of transmission in communities, consistent with emerging evidence (12). Thus, our results differ from the optimal distribution for influenza vaccines, which prioritize school-age children and adults age 30-39 (5). However, the relative susceptibility and infectiousness of SARS-CoV-2 by age remain uncertain. While it is unlikely that susceptibility to infection conditional on exposure is constant across age groups (12), we ran our model to test the sensitivity of this parameter. Under the scenario of constant susceptibility by age, vaccinating those under 20 has a greater impact on reducing cumulative cases than those 2049 (figs. S14 and 15).

Our study is subject to a number of limitations. First, our evaluation strategy focuses on a single country at a time, rather than on between-population allocation (42). Second, we only consider variation in disease severity by age. However, other factors correlate with disease outcomes, such as treatment and healthcare access and comorbidities, which may correlate with factors like rural vs urban location, socioeconomic status, sex $(43,44)$, and race and ethnicity (45), that are not accounted for in this study. Inclusion of these factors in a model would be possible, but only with statistically sound measurements of both their stratified infection risk, contact rates, and disease outcomes. Even in the case of age 
stratification, contact surveys have typically not surveyed those 80 years and older, yet it is this population that suffers dramatically more severe COVID-19 disease and higher infection fatality rates. We extrapolated contact matrices to those older than 80 , but direct measurements would be superior. Last, our study focused on guiding strategy rather than providing more detailed forecasting or estimates (10). As such, we have not made detailed parameter fits to time series of cases or deaths, but rather have used epidemiologic models to identify robust strategies across a range of transmission scenarios.

Our study also considers variation in disease risk only by age, via age-structured contact matrices and age-specific susceptibility, while many discussions around COVID-19 vaccine distribution have thus far focused on prioritizing healthcare or essential workers $(46,47)$. Contact rates, and thus infection potential, vary greatly not only by occupation and age but also by living arrangement (e.g., congregate settings, dormitories), neighborhood and mobility (48-51), and whether the population has a coordinated and fundamentally effective policy to control the virus. With a better understanding of population structure during the pandemic, and risk factors of COVID-19, these limitations could be addressed. Meanwhile, the robust findings in favor of prioritizing those age groups with the highest IFR to minimize mortality could potentially be extended to prioritize those with comorbidities that predispose them to a high IFR, since the strategy of prioritizing the older age groups depends on direct rather than indirect protection.

Vaccine prioritization is not solely a question of science but a question of ethics as well. Hallmarks of the COVID-19 pandemic, as with other global diseases, are inequalities and disparities. While these modeling efforts focus on age and minimizing incidence and death within a simply structured population, other considerations are crucial, from equity in allocation between countries to disparities in access to healthcare, including vaccination, that vary by neighborhood. Thus, the model's simplistic representation of vulnerability (age) should be augmented by better information on the correlates of infection risk and severity. Fair vaccine prioritization should avoid further harming disadvantaged populations. We suggest that, after distribution, pairing serological testing with vaccination in the hardest hit populations is one possible equitable way to extend the benefits of vaccination in settings where vaccination might otherwise not be deemed cost-effective.

\section{REFERENCES AND NOTES}

1. COVID-19 Dashboard by the Center for Systems Science and Engineering at Johns Hopkins University, Online (2020); https://coronavirus. hhu.edu/map.htm.

2. R. Khamsi, If a coronavirus vaccine arrives, can the world make enough? Nature 580, 578-580 (2020). doi:10.1038/d41586-020-01063-8 Medline

3. Framework for equitable allocation of COVID-19 vaccine, Online (2020); www.nap.edu/catalog/25917/framework-for-equitable-allocation-of-covid-19vaccine.

4. D. Weycker, J. Edelsberg, M. E. Halloran, I. M. Longini Jr., A. Nizam, V. Ciuryla, G. Oster, Population-wide benefits of routine vaccination of children against influenza. Vaccine 23, 1284-1293 (2005). doi:10.1016/j,vaccine.2004.08.044 Medline

5. J. Medlock, A. P. Galvani, Optimizing influenza vaccine distribution. Science 325 1705-1708 (2009). doi:10.1126/science. 1175570 Medline

6. S. Bansal, B. Pourbohloul, L. A. Meyers, A comparative analysis of influenza vaccination programs. PLOS Med. 3, e387 (2006). doi:10.1371/journal.pmed.0030387 Medline

7. L. Matrajt, J. Eaton, T. Leung, E. R. Brown, medRxiv (2020). https://www.medrxiv.org/content/10.1101/2020.08.14.20175257v3.

8. M. E. Gallagher et al., medRxiv (2020). https://www.medrxiv.org/content/early/2020/08/11/2020.08.07.20170456.

9. J. H. Buckner, G. Chowell, M. R. Springborn, medRxiv (2020). https://www.medrxiv.org/content/10.1101/2020.09.22.20199174v4.

10. F. Sandmannet al., medRxiv (2020). https://www.medrxiv.org/content/10.1101/2020.09.24.20200857v1.

11. C. J. Worby, H.-H. Chang, Face mask use in the general population and optimal resource allocation during the COVID-19 pandemic. Nat. Commun. 11, 4049 (2020). doi:10.1038/s41467-020-17922-x Medline

12. E. Goldstein, M. Lipsitch, M. Cevik, On the effect of age on the transmission of SARS-CoV-2 in households, schools and the community. J. Infect. Dis. 2020, jiaa691(2020). doi:10.1093/infdis/jiaa691 Medline

13. N. G. Davies, P. Klepac, Y. Liu, K. Prem, M. Jit, R. M. Eggo; CMMID COVID-19 working group. Age-dependent effects in the transmission and control of COVID19 epidemics. Nat. Med. 26, 1205-1211 (2020). doi:10.1038/s41591-020-0962-9 Medline

14. J. Zhang, M. Litvinova, Y. Liang, Y. Wang, W. Wang, S. Zhao, Q. Wu, S. Merler, C. Viboud, A. Vespignani, M. Ajelli, H. Yu, Changes in contact patterns shape the dynamics of the COVID-19 outbreak in China. Science 368, 1481-1486 (2020). doi:10.1126/science. abb8001 Medline

15. S. Herzog et al., medRxiv (2020). https://www.medrxiv.org/content/10.1101/2020.06.08.20125179v3.

16. A. L. Mueller, M. S. McNamara, D. A. Sinclair, Why does COVID-19 disproportionately affect older people? Aging (Albany NY) 12, 9959-9981 (2020). doi:10.18632/aging.103344 Medline

17. Y. Liu, B. Mao, S. Liang, J.-W. Yang, H.-W. Lu, Y.-H. Chai, L. Wang, L. Zhang, Q.-H. Li, L. Zhao, Y. He, X.-L. Gu, X.-B. Ji, L. Li, Z.-J. Jie, Q. Li, X.-Y. Li, H.-Z. Lu, W.-H. Zhang, Y.-L. Song, J.-M. Qu, J.-F. Xu; Shanghai Clinical Treatment Experts Group for COVID-19, Association between age and clinical characteristics and outcomes of COVID-19. Eur. Respir. J. 55, 2001112 (2020). doi:10.1183/13993003.01112-2020 Medline

18. J. Westmeier et al., mBio 11, e02243-20 (2020).

19. A. T. Levin, W. P. Hanage, N. Owusu-Boaitey, K. B. Cochran, S. P. Walsh, G. Meyerowitz-Katz, Assessing the age specificity of infection fatality rates for COVID-19: Systematic review, meta-analysis, and public policy implications. Eur. J. Epidemiol. 35, 1123-1138 (2020). doi:10.1007/s10654-020-00698-1 Medline

20. H. Salje, C. Tran Kiem, N. Lefrancq, N. Courtejoie, P. Bosetti, J. Paireau, A. Andronico, N. Hozé, J. Richet, C.-L. Dubost, Y. Le Strat, J. Lessler, D. Levy-Bruhl, A. Fontanet, L. Opatowski, P.-Y. Boelle, S. Cauchemez, Estimating the burden of SARS-CoV-2 in France. Science 369, 208-211(2020). doi:10.1126/science. abc3517 Medline

21. J. K. H. Lee, G. K. L. Lam, T. Shin, J. Kim, A. Krishnan, D. P. Greenberg, A. Chit, Efficacy and effectiveness of high-dose versus standard-dose influenza vaccination for older adults: A systematic review and meta-analysis. Expert Rev. Vaccines 17, 435-443 (2018). do::10.1080/14760584.2018.1471989 Medline 
22. T. M. E. Govaert, C. T. Thijs, N. Masurel, M. J. Sprenger, G. J. Dinant, J. A. Knottnerus, The efficacy of influenza vaccination in elderly individuals. A randomized double-blind placebo-controlled trial. JAMA 272, 1661-1665(1994). doi:10.1001/jama.1994.03520210045030 Medline

23. J. A. Lewnard, S. Cobey, Immune History and Influenza Vaccine Effectiveness. Vaccines (Basel) 6, 28 (2018). doi:10.3390/vaccines6020028 Medline

24. S. F. Lumley et al., N. Engl. J. Med. 10.1056/NEJMoa2034545 (2020).

25. City of New York, COVID-19 data, (2020); wwwlnyc.gov/site/doh/covid/covid19-data-testing.page.

26. K. L. Bajema et al., JAMA Intern. Med. 10.1001/jamainternmed.2020.7976 (2020).

27. H. Ward et al., medRxiv (2020) https://www.medrxiv.org/content/10.1101/2020.10.26.20219725v1.

28. J. M. Dan et al., bioRxiv (2020). https://www.biorxiv.org/content/10.1101/2020.11.15.383323v2

29. D. B. Larremore et al., medRxiv (2020) https://www.medrxiv.org/content/10.1101/2020.04.15.20067066v2.

30. D. Ellenberger, R. A. Otten, B. Li, M. Aidoo, I. V. Rodriguez, C. A. Sariol, M. Martinez, M. Monsour, L. Wyatt, M. G. Hudgens, E. Kraiselburd, B. Moss, H. Robinson, T. Folks, S. Butera, HIV-1 DNA/MVA vaccination reduces the per exposure probability of infection during repeated mucosal SHIV challenges. Virology 352, 216-225 (2006). doi:10.1016/j.virol.2006.04.005 Medline

31. K. E. Langwig, A. R. Wargo, D. R. Jones, J. R. Viss, B. J. Rutan, N. A. Egan, P. SáGuimarães, M. S. Kim, G. Kurath, M. G. M. Gomes, M. Lipsitch, Vaccine Effects on Heterogeneity in Susceptibility and Implications for Population Health Management. mBio 8, e00796-17 (2017). doi:10.1128/mBio.00796-17 Medline

32. H. R. Sharpe, C. Gilbride, E. Allen, S. Belij-Rammerstorfer, C. Bissett, K. Ewer, T. Lambe, The early landscape of coronavirus disease 2019 vaccine development in the UK and rest of the world. Immunology 160, 223-232 (2020). doi:10.1111/imm.13222 Medline

33. M. Kornfield, Washington Post (2020). https://www.washingtonpost.com/health/2020/12/02/kids-vaccine-delay/.

34. K. Premet al., medRxiv (2020). https://www.medrxiv.org/content/10.1101/2020.07.22.20159772v2.

35. C. I. Jarvis, K. Van Zandvoort, A. Gimma, K. Prem, P. Klepac, G. J. Rubin, W. J. Edmunds; CMMID COVID-19 working group, Quantifying the impact of physical distance measures on the transmission of COVID-19 in the UK. BMC Med. 18, 124 (2020). doi:10.1186/s12916-020-01597-8 Medline

36. J. A. Backer et al., medRxiv (2020) https://www.medrxiv.org/content/10.1101/2020.05.18.20101501v2

37. M. Brenan, Willingness to Get COVID-19 Vaccine Ticks Up to 63\% in U.S., Online (December 8, 2020). https://news.gallup.com/poll/327425/willingness-covidvaccine-ticks.aspx.

38. S. Ghisolfiet al., Center for Global Development (2020).

39. M. Lipsitch, N. E. Dean, Understanding COVID-19 vaccine efficacy. Science 370 , 763-765 (2020). doi:10.1126/science.abe5938 Medline

40. L. E. Duijzer, W. L. van Jaarsveld, J. Wallinga, R. Dekker, Dose-Optimal Vaccine Allocation over Multiple Populations. Prod. Oper. Manag. 27, 143-159 (2018). doi:10.1111/poms.12788 Medline

41. T. Takahashi, M. K. Ellingson, P. Wong, B. Israelow, C. Lucas, J. Klein, J. Silva, T. Mao, J. E. Oh, M. Tokuyama, P. Lu, A. Venkataraman, A. Park, F. Liu, A. Meir, J. Sun, E. Y. Wang, A. Casanovas-Massana, A. L. Wyllie, C. B. F. Vogels, R. Earnest, S. Lapidus, I. M. Ott, A. J. Moore, A. Shaw, J. B. Fournier, C. D. Odio, S. Farhadian, C. Dela Cruz, N. D. Grubaugh, W. L. Schulz, A. M. Ring, A. I. Ko, S. B. Omer, A. Iwasaki; Yale IMPACT Research Team, Sex differences in immune responses that underlie COVID-19 disease outcomes. Nature 588, 315-320 (2020). doi:10.1038/s41586-020-2700-3 Medline

42. D. Chakravarty, S. S. Nair, N. Hammouda, P. Ratnani, Y. Gharib, V. Wagaskar, N. Mohamed, D. Lundon, Z. Dovey, N. Kyprianou, A. K. Tewari, Sex differences in SARS-CoV-2 infection rates and the potential link to prostate cancer. Commun. Biol. 3, 374 (2020). doi:10.1038/s42003-020-1088-9 Medline

43. M. Webb Hooper, A. M. Nápoles, E. J. Pérez-Stable, COVID-19 and Racial/Ethnic Disparities. JAMA 323, 2466-2467 (2020). doi:10.1001/jama.2020.8598 Medline
44. M. Jenco, AAP News (2020)

https://www.aappublications.org/news/2020/08/27/covid19vaccinepriorities 082620.

45. J. Cohen, The line starts to form for a coronavirus vaccine. Science $369,15-16$ (2020). doi:10.1126/science.369.6499.15 Medline

46. S. Mishra, J. C. Kwong, A. K. Chan, S. D. Baral, Understanding heterogeneity to inform the public health response to COVID-19 in Canada. CMAJ 192, E684E685 (2020). doi:10.1503/cmaj.201112 Medline

47. L. Hawks, S. Woolhandler, D. McCormick, COVID-19 in Prisons and Jails in the United States. JAMA Intern. Med. 180, 1041-1042 (2020). doi:10.1001/jamainternmed.2020.1856 Medline

48. H. S. Badr et al., Lancet Infect. Dis. 10.1016/S1473-3099(20)30861-6 (2020).

49. J. Ducharme, Time (2020); https://time.com/5870041/COVID-19neighborhood-risk

50. R Core Team, R: A Language and Environment for Statistical Computing, $\mathrm{R}$ Foundation for Statistical Computing, Vienna, Austria (2019)

51. K. E. Atkinson, An Introduction to Numerical Analysis (Wiley, New York, 1989), chap. 2, pp. 56-58, second edn.

52. P. A. Gross, A. W. Hermogenes, H. S. Sacks, J. Lau, R. A. Levandowski, The efficacy of influenza vaccine in elderly persons. A meta-analysis and review of the literature. Ann. Intern. Med. 123, 518-527 (1995). doi:10.7326/0003-4819123-7-199510010-00008 Medline

53. P. Span, New York Times (2020). https://www.nytimes.com/2020/06/19/health/vaccine-trials-elderly.html.

54. K. M. Bubar et al., COVID-19 vaccine prioritization code. Zenodo (2020). doi:10.5281/zenodo.4308794.

55. W. G. H. Observatory, Life tables by country, (2016); https://apps. who.int/gho/data/view.main.LT62160?lang=en.

56. U. N. D. of Economic, S. A. P. Division, World population prospects (2019); https://population.un.org/wpp

57. C. H. Geurtsvan Kesselet al., Nat. Commun. 11, 3436 (2020).

\section{ACKNOWLEDGMENTS}

The authors wish to thank Sereina Herzog, Mark Jit, Jacco Wallinga, and Helen Johnson for their feedback. Funding: KMB was supported in part by the Interdisciplinary Quantitative Biology (IQ Biology) PhD program at the BioFrontiers Institute, University of Colorado Boulder. KMB and DBL were supported in part through the MIDAS Coordination Center (MIDASNI2020-2) by a grant from the National Institute of General Medical Science (3U24GM13201302S2). ML, SMK, and YHG were supported in part by the Morris-Singer Fund for the Center for Communicable Disease Dynamics at the Harvard T.H. Chan School of Public Health. ML and DBL were supported in part by the SeroNet program of the National Cancer Institute (1U01CA261277-01). Author Contributions: KMB, SMK, ML, SC, YHG and DBL conceived of the study. KMB and DBL performed the analyses. KMB and KR generated all figures. KR created interactive visualization tools. All authors wrote and revised the manuscript. Competing Interests:ML discloses honoraria/consulting from Merck, Affinivax, Sanofi-Pasteur, Bristol Myers Squibb, and Antigen Discovery; research funding (institutional) from Pfizer; an unpaid scientific advice to Janssen, Astra-Zeneca, and Covaxx (United Biomedical); and is an Honorary Faculty Member, Wellcome Sanger Institute, and an Associate Member, Broad Institute. YHG discloses consulting for Merck and GlaxoSmithKline, and research funding from Pfizer not related to this project or topic. DBL is a member of the scientific advisory board of Darwin BioSciences. Data and materials availability: Reproduction code is open source and provided by the authors (54). This work is licensed under a Creative Commons Attribution 4.0 International (CC BY 4.0) license, which permits unrestricted use, distribution, and reproduction in any medium, provided the original work is properly cited. To view a copy of this license, visit https://creativecommons.org/licenses/by/4.0/. This license does not apply to figures/photos/artwork or other content included in the article that is credited to a third party; obtain authorization from the rights holder before using such material. 


\section{SUPPLEMENTARY MATERIALS}

science.sciencemag.org/cgi/content/full/science.abe6959/DC1

Materials and Methods

Supplementary Text

Figs. S1 to S15

Tables S1 and S2

References (55-57)

MDAR Reproducibility Checklist

8 September 2020; accepted 12 January 2021

Published online 21 January 2021

10.1126/science.abe6959 

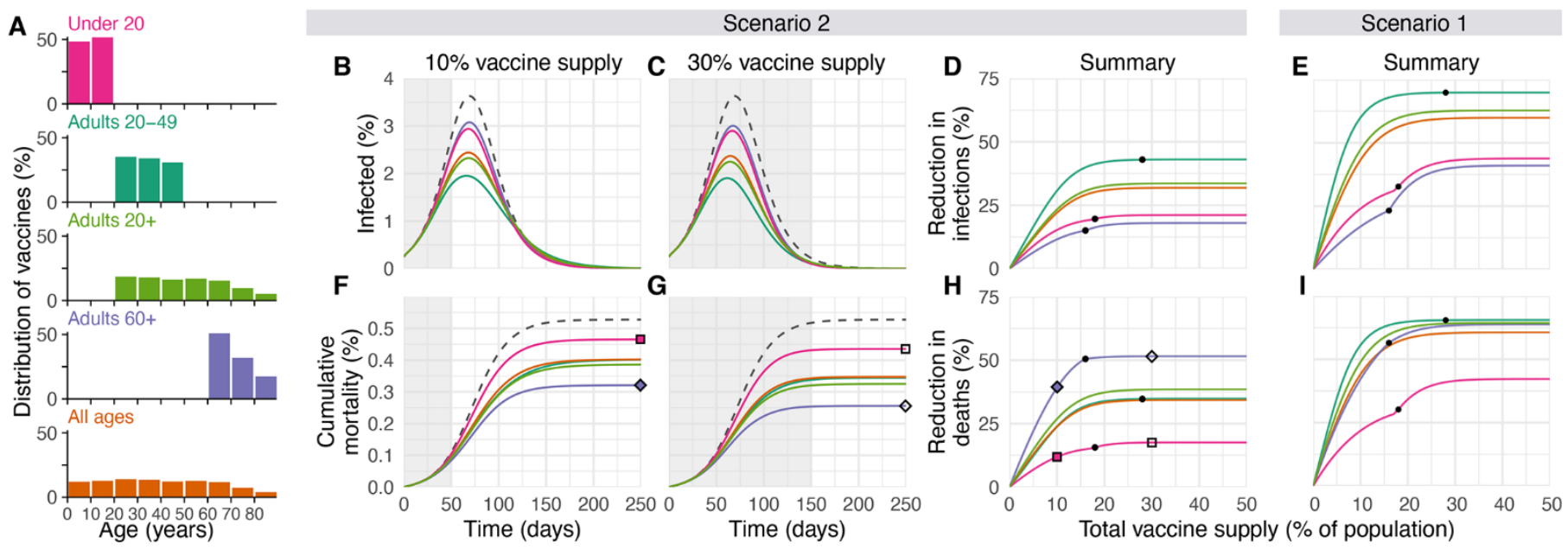

Fig. 1. Impacts of vaccine prioritization strategies on mortality and infections. (A) Distribution of vaccines for five prioritization strategies: under 20, adults 20-49, adults 20+, adults 60+ and all ages. (B and C) Example simulation curves show percentage of the total population infected over time and ( $F$ and $G$ ) cumulative mortality for no vaccines (grey dashed lines) and for five different prioritization strategies [colored lines matching (A)], with 10\% [(B) and (F)] and 30\% [(C) and (G)] vaccine supply. Summary curves show percent reductions in (D and $\mathrm{E}$ ) infections and ( $\mathrm{H}$ and $\mathrm{I})$ deaths in comparison to an unmitigated outbreak for vaccine supplies between $1 \%$ and $50 \%$ after 365 days of simulation. Squares and diamonds show how the outputs from single simulations $[(F)$ and $(G)]$ correspond to points in summary curves $(H)$. Grey shading indicates period during which vaccine is being rolled out at $0.2 \%$ of total population per day. Black dots indicate breakpoints at which prioritized demographic groups have been $70 \%$ vaccinated, after which vaccines are distributed without prioritization. These simulations assume contact patterns and demographics of the United States $(37,52)$ and an all-ornothing, transmission-blocking vaccine with $90 \%$ vaccine efficacy and $\left.R_{0}=1.5\right)$ (Scenario 2$)$ and $\left(R_{0}=1.15\right.$ ) (Scenario 1). 


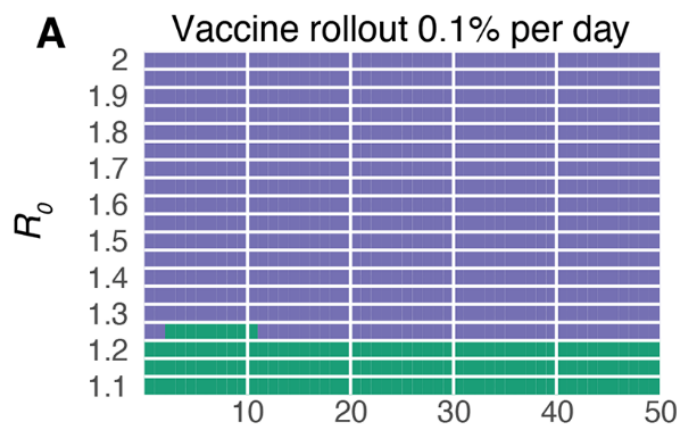

B Vaccine rollout $0.2 \%$ per day
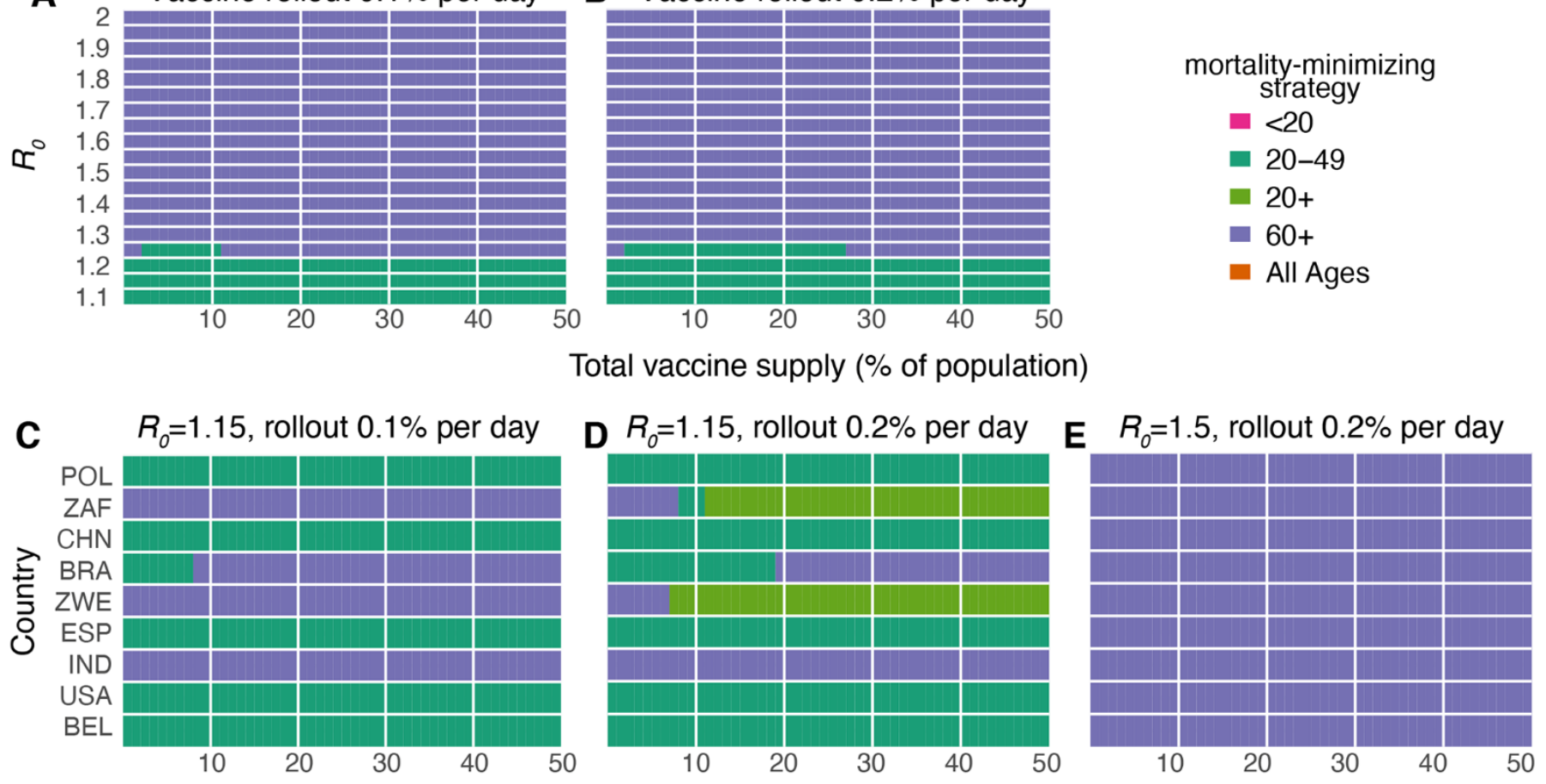

Total vaccine supply (\% of population)

Fig. 2. Mortality-minimizing vaccine prioritization strategies across reproductive numbers $R_{0}$ and countries. Heatmaps show the prioritization strategies resulting in maximum reduction of mortality for varying values of the basic reproductive number $R_{0}(A$ and $B)$ and across nine countries $(C, D$, and $E)$, for vaccine supplies between $1 \%$ and $50 \%$ of the total population, for an all-or-nothing and transmission blocking vaccine, $90 \%$ vaccine efficacy. (A, B) Shown: contact patterns and demographics of the United States (37, 52); [(C), (D), and (E)] Shown: contact patterns and demographics of POL, Poland; ZAF, South Africa; CHN, China; BRA, Brazil; ZWE, Zimbabwe; ESP, Spain; IND, India; USA, United States of America; $\mathrm{BEL}$, Belgium, with $R_{0}$ and rollout speeds as indicated. 

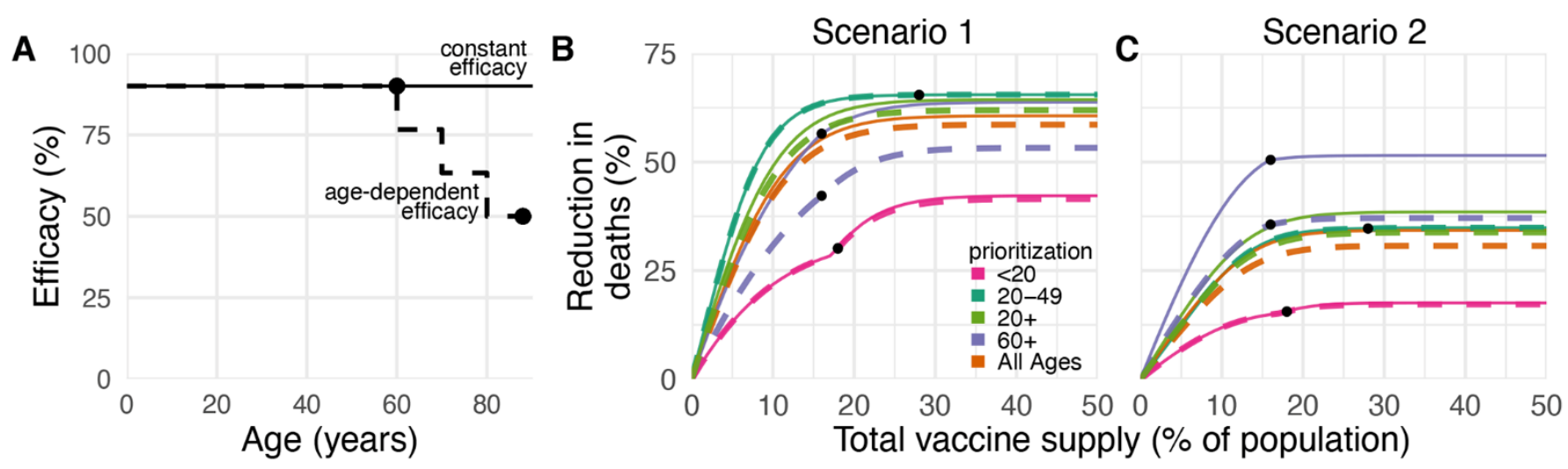

Fig. 3. Effects of age-dependent vaccine efficacy on the impacts of prioritization strategies. (A) Diagram of hypothetical age-dependent vaccine efficacy shows decrease from $90 \%$ baseline efficacy to $50 \%$ efficacy among individuals $80+$ beginning at age 60 (dashed line). (B and C) Percent reduction in deaths in comparison to an unmitigated outbreak for transmission-blocking all-or-nothing vaccines with either constant $90 \%$ efficacy for all age groups (solid lines) or age-dependent efficacy shown in (A) (dashed lines), covering Scenario 1 [0.2\% rollout/day, $\left.R_{0}=1.15 ;(B)\right]$ and Scenario $2\left[0.2 \%\right.$ rollout/day, $\left.R_{0}=1.5(\mathrm{C})\right]$. Black dots indicate breakpoints at which prioritized demographic groups have been $70 \%$ vaccinated, after which vaccines are distributed without prioritization. Shown: contact patterns and demographics of the United States (37, 52); all-or nothing and transmission blocking vaccine. 

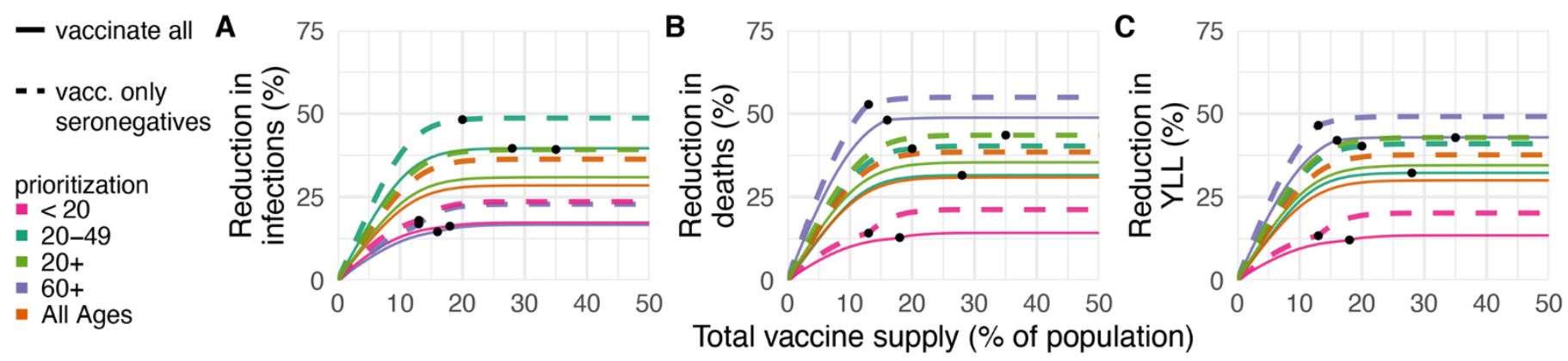

Fig. 4. Effects of existing seropositivity on the impacts of prioritization strategies. Percent reductions in (A) infections, (B) deaths, and (C) years of life lost (YLL) for prioritization strategies when existing age-stratified seroprevalence is incorporated [August 2020 estimates for New York City; mean seroprevalence 26.9\% (27)]. Plots show reductions for Scenario $2\left(0.2 \%\right.$ rollout/day, $\left.R_{0}=1.5\right)$ when vaccines are given to all individuals (solid lines) or to only seronegatives (dashed lines), inclusive of $96 \%$ serotest sensitivity, $99 \%$ specificity (53), and approximately three months of seroreversion (supplementary materials, materials and methods) (29). Shown: U.S. contact patterns and demographics $(37,52)$; all-or-nothing and transmission-blocking vaccine with $90 \%$ vaccine efficacy. See figs. S12 and S13 for lower and higher seroprevalence examples, respectively. 